\title{
Homogeneous Metrics on Spheres
}

\author{
Martin Kerin AND David Wraith
}

This article is a summary of the work carried out by the first author towards a Master's thesis under the direction of the second author.

Our aim is to investigate certain aspects of the geometry of spheres, especially the spheres $S^{3}$ and $S^{5}$. Usually, when one imagines a sphere, one imagines a round sphere, and of course the geometry of such objects is well understood. However, there are many different geometries with which a sphere can be equipped.

A sphere is first and foremost a smooth manifold. It only becomes a geometric object - in other words assumes some kind of shape (such as being round) - when we equip it with a Riemannian metric. Recall that a Riemannian metric is a smoothly varying choice of inner product on each tangent space. Of course there are uncountably many Riemannian metrics we can equip any sphere with, and each will provide the sphere with a certain geometry. However, we will focus on certain very special kinds of Riemannian metrics, namely those which are homogeneous.

A round sphere is clearly highly symmetric. More than just having lots of symmetry, it in fact looks the same at every point! Spaces with this property are known as homogeneous spaces. More formally, if $M$ is a smooth manifold, we say that $M$ is a homogeneous space if there is a (Lie) group $G$ of self-diffeomorphisms of $M$ which acts transitively. In other words, given any two points $x$ and $y$ in $M$, there is an element $g \in G$ such that $g x=y$.

Any homogeneous space $M$ can be regarded as a space of cosets in the following way. Choose a point $x \in M$. Let $H$ be the subgroup of $G$ which fixes the point $x$ (that is, $h x=x$ for all $h \in H$ ). $H$ is called the isotropy subgroup of $G$ at the point $x$. (The isotropy at any other point in $M$ is conjugate in $G$ to $H$.) It is not difficult 
to establish that the space of cosets $G / H$ can be equipped with a smooth structure so that $G / H$ is diffeomorphic to $M$. For any choice of $x \in M$, an explicit diffeomorphism is given by $g H \mapsto g x$.

Spheres can be expressed as homogeneous spaces in many different ways. Here is a complete list:

$$
\begin{gathered}
\frac{S O(n+1)}{S O(n)} \cong S^{n} ; \\
\frac{U(n+1)}{U(n)} \cong \frac{S U(n+1)}{S U(n)} \cong S^{2 n+1} ; \\
\frac{S p(n+1) S p(1)}{S p(n) S p(1)} \cong \frac{S p(n+1) U(1)}{S p(n) U(1)} \cong \frac{S p(n+1)}{S p(n)} \cong S^{4 n+1} ; \\
\frac{G_{2}}{S U(3)} \cong S^{6} ; \quad \frac{S p i n_{7}}{G_{2}} \cong S^{7} ; \quad \frac{\operatorname{Spin}_{9}}{\operatorname{Spin}_{7}} \cong S^{15} .
\end{gathered}
$$

Here $\cong$ denotes diffeomorphism. See $[\mathbf{1} ;$ p. 179] for details.

In our discussion of homogeneous spaces we have so far said nothing about geometry. For any homogeneous space $G / H$ there are natural geometries to consider. These are the geometries which are invariant under the transitive symmetry group $G$. We define a homogeneous metric on $G / H$ to be a Riemannian metric $\langle$,$\rangle satisfying$

$$
\left\langle g_{*} v, g_{*} w\right\rangle_{g x}=\langle v, w\rangle_{x}
$$

where $v, w$ are tangent vectors at $x \in G / H$, and $g_{*}$ denotes the derivative of the diffeomorphism $g \in G$. A homogeneous space equipped with such a metric is called a Riemannian homogeneous space.

We will show that different descriptions of the sphere as a homogeneous space give rise to different families of homogeneous metrics, and hence to different natural geometries. In order to better understand the geometries that arise we will compute the curvatures of our metrics. Recall that the round metric has constant (sectional) curvature, and is the unique metric up to scaling with this property. Of course, before we can calculate curvatures, we must first identify and describe these homogeneous metrics.

We will explain how to construct any homogeneous metric in two different ways. We will need both. As a preliminary, though, we must describe the isotropy and adjoint representations. 
Consider a homogeneous space $G / H$. This space has a distinguished point, namely the identity coset $e H$. Clearly $H$ is the isotropy subgroup at this point. As each element $h \in H$ fixes $e H$, its derivative $h_{*}$ must therefore be a linear automorphism of the tangent space $T_{e H} G / H$. Thus we get a representation

$$
\rho: H \longrightarrow \operatorname{Aut}\left(T_{e H} G / H\right)
$$

called the isotropy representation.

Next we turn our attention to the (Lie) group $G$. There is a group homomorphism $I: H \rightarrow \operatorname{Diff}(G)$ given by $I(h)(g)=h g h^{-1}$. (Here, $\operatorname{Diff}(G)$ is the group of self-diffeomorphisms of $G$.) If we differentiate $I$ at the identity $e \in G$, we obtain the adjoint representation

$$
\operatorname{Ad}_{H}: H \longrightarrow \operatorname{Aut}\left(T_{e} G\right) .
$$

To return to homogeneous metrics on $G / H$, suppose we choose an inner product on the tangent space $T_{e H} G / H$. Using the (left) action of $G$ on $G / H$, we can attempt to propagate this inner product around $G / H$ to produce a Riemannian metric. Specifically, at the coset $g H$ we introduce the inner product

$$
\langle v, w\rangle_{g H}:=\left\langle g_{*}^{-1} v, g_{*}^{-1} w\right\rangle_{e H} .
$$

However, such a Riemannian metric is not necessarily well-defined. In order to avoid this problem it is necessary and sufficient for the original inner product at $e H$ to be invariant under the isotropy representation. Therefore for each such inner product we obtain a Riemannian metric on our homogeneous space by propagation. Moreover, it is immediate that the resulting metric is homogeneous. It is equally easy to see that any homogeneous metric can be constructed in this way.

As an alternative approach to constructing homogeneous metrics, we can begin by considering Riemannian metrics on the group $G$, and ask when such a metric induces a well-defined homogeneous metric on passing to the quotient $G / H$. We require the following set-up. Denote the tangent space $T_{e} G$ by $\mathbf{g}$, and the subspace of $\mathbf{g}$ tangent to $H$ by $\mathbf{h}$. Let $\mathbf{m}$ be a complement to $\mathbf{h}$ in $\mathbf{g}$ - in other words $\mathbf{g}=\mathbf{m} \oplus \mathbf{h}$. Using the (derivative of) the left action of $G$ on itself, we can propagate $\mathbf{m}$ to every point of $G$, giving us a distribution of 
tangent subspaces $\left\{\mathbf{m}_{g}\right\}_{g \in G}$. Notice that for every $g \in G$ we then have a vector space isomorphism

$$
\mathbf{m}_{g} \cong T_{g H} G / H
$$

given by the derivative $\pi_{*}$ of the quotient map $\pi: G \rightarrow G / H$. Thus a smoothly varying choice of inner product on the distribution $\left\{\mathbf{m}_{g}\right\}_{g \in G}$ gives rise to a Riemannian metric on $G / H$. Again we must address the question of when such a metric is well-defined, and when it is homogeneous. Suppose that the space $\mathbf{m}$ is $\mathrm{Ad}_{H}$-invariant. All groups under consideration in this paper will be compact, and under these conditions we can always find such an $\mathbf{m}$. Furthermore, we can always suppose that $\mathbf{m}$ is equipped with an $\mathrm{Ad}_{H}$-invariant inner product. Then propagating this inner product around the distribution $\left\{\mathbf{m}_{g}\right\}_{g \in G}$ gives rise to an inner product on $G / H$ which is both well-defined and homogeneous. In particular, the induced inner product at $T_{e H} G / H$ is isotropy invariant. It is not difficult to show that any homogeneous metric on $G / H$ can be constructed in this way.

Note that we can construct Riemannian metrics on $G$ starting with any inner product on $\mathbf{g}$ and propagating by the left action of $G$ on itself. Such metrics are called left invariant. Given an $\mathrm{Ad}_{H}$-invariant inner product on $\mathbf{m}$ as above, we extend to an inner product on the whole of $\mathbf{g}$ by choosing an arbitrary inner product on $\mathbf{h}$ and declaring $\mathbf{m}$ and $\mathbf{h}$ to be orthogonal. The resulting left invariant metric on $G$ not only induces a well-defined homogeneous metric on $G / H$, but makes $\pi: G \rightarrow G / H$ into a Riemannian submersion. For definitions and details about Riemannian submersions. see $[\mathbf{1} ;$ ch. 9]. The fact that we get a Riemannian submersion is significant for our purposes because there are well-known formulas due to O'Neill $[\mathbf{1} ;$ ch. $9, \S \mathrm{D}]$ which help in the calculation of curvature for such objects. We will return to the O'Neill formulas later on.

We now know how to construct homogeneous metrics, but for a given description of a sphere as a homogeneous space, we want to be able to find all such metrics. The key to being able to do this is as follows.

Consider a group homomorphism

$$
\theta: G \longrightarrow \operatorname{Aut}(V)
$$

where $V$ is a finite dimensional real vector space. (In other words, $\theta$ is a representation of $G$ on $V$.) Furthermore, suppose that this 
representation is irreducible. This means that $V$ has no proper $G$ invariant subspace. Then up to scaling by a positive constant, there is a unique $G$-invariant inner product on $V$. (This assertion is not difficult to prove, and is essentially a consequence of Schur's Lemma.)

Let us explore the consequences of this result for homogeneous spaces. If the isotropy representation at $e H$ is irreducible, then it follows that there is a unique isotropy-invariant inner product on $T_{e H} G / H$ up to scaling, and hence a unique homogeneous metric on $G / H$, again up to a global (positive!) scaling factor. Suppose now that $T_{e H} G / H$ is not isotropy irreducible, but splits into a direct sum of irreducible subspaces. Then on each of these subspaces we must have a unique isotropy-invariant inner product up to scaling. Of course the various scaling factors are completely independent of each other. Having an invariant inner product on such a collection of subspaces does not in itself determine an invariant inner product on the whole space (for example we know nothing of the angles between subspaces). However, if these sub-representations are inequivalent, then it can be shown that for any invariant inner product on the whole space, the invariant subspaces must all be mutually orthogonal. This in fact will be sufficient to determine the complete set of invariant metrics for the cases we study below.

We begin our study of the homogenous geometry of spheres by looking at

$$
\frac{S O(n+1)}{S O(n)} \cong S^{n}
$$

We will assume $n \geq 2$. Think of $S^{n}$ as $\left\{\left(x_{1}, \ldots, x_{n+1}\right) \mid \sum_{i=1}^{n} x_{i}^{2}=1\right\}$ $\subset \mathbb{R}^{n+1}$. Expressing points in $S^{n}$ as column vectors (with respect to the standard basis), the transitive action of $S O(n+1)$ is then given by multiplying column vectors from the left. (In future we will refer to the action defined by left multiplication of column vectors as the natural action.) Notice that the isotropy group of the point $(1,0, \ldots 0) \in S^{n}$ is precisely the collection of matrices of the form

$$
\left(\begin{array}{ll}
1 & \\
& S O(n)
\end{array}\right) \subset S O(n+1)
$$

and this subgroup is clearly canonically isomorphic to $S O(n)$. The isotropy representation is the derivative of the natural action restricted to the isotropy subgroup, and it is easy to see that this representation is equivalent to the standard representation of $S O(n)$ 
on $\mathbb{R}^{n}$. Since $S O(n)$ is the full group of rotations of $\mathbb{R}^{n}$, it is clear that there can be no invariant subspaces. In other words, our isotropy representation must be irreducible. By the above it follows that there is a unique invariant inner product on $T_{(1,0 \ldots 0)} S^{n}$ up to scaling by a positive constant, and hence there is a unique homogeneous metric on $\frac{S O(n+1)}{S O(n)} \cong S^{n}$ up to scaling by a positive constant. It remains to identify this one-parameter family of metrics. However, this is not difficult as the round metric (of any radius) is clearly invariant under the natural action of $S O(n+1)$ - that is, invariant under all rotations. Therefore the round metrics of all possible radii are precisely the homogeneous metrics for $\frac{S O(n+1)}{S O(n)}$. For contrast with later results, recall that the round metric on $S^{n}$ of radius $R$ has constant sectional curvature of $\frac{1}{R^{2}}$, constant Ricci curvature (for unit vectors) of $\frac{n-1}{R^{2}}$ and constant scalar curvature $\frac{n(n-1)}{R^{2}}$.

We now turn our attention to a case with a much larger family of homogeneous metrics: $\frac{S U(2)}{S U(1)} \cong S^{3}$. Note that $S U(1)$ is the trivial group, and hence the collection of homogeneous metrics on this homogeneous space is precisely the set of left-invariant metrics on $S U(2) \cong S^{3}$. This last diffeomorphism can be seen clearly from the following definition of $S U(2)$ :

$$
S U(2)=\left\{\left(\begin{array}{cc}
z & w \\
-\bar{w} & \bar{z}
\end{array}\right): z, w \in \mathbb{C},|z|^{2}+|w|^{2}=1\right\} .
$$

As described above, the left-invariant metrics on a Lie group are in one-to-one correspondance with inner products at the identity. Hence this is a very large family, and we will content ourselves with studying some special cases.

First, though, we address the problem of computing the curvature of left-invariant metrics on Lie groups. (The curvature formulas for bi-invariant metrics on Lie groups is both well-known and simple, see $[2 ;$ p. 57].)

As a preliminary we introduce the Lie-bracket operation. If $X$ and $Y$ are any smooth tangent vector fields on a manifold $M$, the Lie bracket of $X$ and $Y$,

$$
[X, Y]:=X Y-Y X
$$


is another tangent vector field, and this gives the space of tangent vector fields on $M$ an algebra structure. More precisely, it makes it into a Lie algebra. If our manifold happens to be a Lie group, and $X$ and $Y$ are left-invariant vector fields, it can be shown that $[X, Y]$ is also left-invariant. As any left-invariant vector field is determined by its vector at the identity, we obtain a Lie algebra structure on the tangent space to the identity. In the special case of a matrix group, the tangent space at the identity can be identified with a space of matrices, and the Lie bracket operation turns out to be $[A, B]=A B-B A$, where $A$ and $B$ are matrices from the tangent space and the expression $A B-B A$ is given by matrix multiplication.

Next note that since we are considering left-invariant metrics, the curvatures we obtain must also display left-invariance and therefore it suffices to calculate these at the identity. For definitions of the various kinds of curvature we will investigate, see $[\mathbf{2} ;$ ch. 2]. Our approach is to use the Koszul formula [2; p. 22] to compute the Levi-Civita connection, and from there to compute the curvature tensor directly. We obtain the following results. Let $X_{1}, \ldots, X_{n}$ be an orthonormal basis for the tangent space to the identity. We will use the symbol $c_{i j}^{k}$ to denote the quantity $\left\langle\left[X_{i}, X_{j}\right], X_{k}\right\rangle$. The sectional curvature of the plane spanned by $X_{i}$ and $X_{j}$ is then given by

$$
\begin{aligned}
K\left(X_{i}, X_{j}\right)= & -\frac{3}{4} \sum_{k}\left(c_{i j}^{k}\right)^{2}+\frac{1}{4} \sum_{k}\left(c_{i k}^{j}\right)^{2}+\frac{1}{4} \sum_{k}\left(c_{j k}^{i}\right)^{2}-\sum_{k} c_{i k}^{i} c_{j k}^{j} \\
& +\frac{1}{2} \sum_{k} c_{i j}^{k}\left(c_{k i}^{j}-c_{k j}^{i}\right)+\frac{1}{2} \sum_{k} c_{j k}^{i} c_{i k}^{j} .
\end{aligned}
$$

The Ricci and scalar curvatures can then be obtained by taking averages of sectional curvatures.

Returning now to left-invariant metrics on $S U(2)$, note that the tangent space at the identity (the Lie algebra $s u(2)$ of $S U(2)$ ) can be identified with the (real) vector space of all two-by-two skewhermitian matrices with trace zero. A basis is given by

$$
e_{1}=\left(\begin{array}{rr}
i & 0 \\
0 & -i
\end{array}\right), \quad e_{2}=\left(\begin{array}{rr}
0 & 1 \\
-1 & 0
\end{array}\right), \quad e_{3}=\left(\begin{array}{cc}
0 & i \\
i & 0
\end{array}\right) .
$$

This basis is orthonormal for the inner product

$$
\langle A, B\rangle=-\frac{1}{2} \operatorname{tr}(A B)
$$


(That this expression defines an inner product follows from the fact that it is a scaling of the Killing form of $s u(2)$, and $S U(2)$ is a compact semi-simple group.) Denote by $g$ the left-invariant metric on $S U(2)$ determined by $\langle$,$\rangle .$

Consider the scaled left-invariant metric $g_{\mu}$, which on $s u(2)$ is given by

$$
g_{\mu}\left(e_{i}, e_{j}\right)=\left\{\begin{array}{c}
\mu_{1} \text { if } i=j=1 \\
\mu_{2} \text { if } i=j=2 \\
\mu_{3} \text { if } i=j=3 \\
0 \text { otherwise. }
\end{array}\right.
$$

Note that $\left\{e_{i}\right\}$ is not an orthonormal set for $g_{\mu}$ ! However, $\left\{\epsilon_{i}\right\}$ where $\epsilon_{i}:=\frac{1}{\sqrt{\mu_{i}}} e_{i}$ is orthonormal for $g_{\mu}$. Using the above left-invariant curvature calculations we find that

$$
\begin{aligned}
& K\left(\epsilon_{1}, \epsilon_{2}\right)=-3 \frac{\mu_{3}}{\mu_{1} \mu_{2}}+\frac{\mu_{2}}{\mu_{1} \mu_{3}}+\frac{\mu_{1}}{\mu_{2} \mu_{3}}+\frac{2}{\mu_{1}}+\frac{2}{\mu_{2}}-\frac{2}{\mu_{3}} \\
& K\left(\epsilon_{1}, \epsilon_{3}\right)=-3 \frac{\mu_{2}}{\mu_{1} \mu_{3}}+\frac{\mu_{3}}{\mu_{1} \mu_{2}}+\frac{\mu_{1}}{\mu_{2} \mu_{3}}+\frac{2}{\mu_{1}}+\frac{2}{\mu_{3}}-\frac{2}{\mu_{2}} \\
& K\left(\epsilon_{2}, \epsilon_{3}\right)=-3 \frac{\mu_{1}}{\mu_{2} \mu_{3}}+\frac{\mu_{3}}{\mu_{1} \mu_{2}}+\frac{\mu_{2}}{\mu_{1} \mu_{3}}+\frac{2}{\mu_{2}}+\frac{2}{\mu_{3}}-\frac{2}{\mu_{1}} .
\end{aligned}
$$

Observe the symmetry in these formulas. Clearly, by choosing the constants $\mu_{i}$ suitably, we can arrange for $g_{\mu}$ to be very different from a round metric.

To help analyse these formulas, let us consider the very special case where $\mu_{1}=\lambda$ (a positive constant), and $\mu_{2}=\mu_{3}=1$. Here we have

$$
\begin{gathered}
K\left(\epsilon_{1}, \epsilon_{2}\right)=\lambda ; \\
K\left(\epsilon_{1}, \epsilon_{3}\right)=\lambda ; \\
K\left(\epsilon_{2}, \epsilon_{3}\right)=4-3 \lambda .
\end{gathered}
$$

The significance of this family of metrics is as follows. $S^{3}$ admits a Hopf fibration. That is, $S^{3}$ is the total space of a fibre bundle with fibre $S^{1}$ and base $S^{2}$. More precisely, consider the standard (unit radius) $S^{3} \subset \mathbb{C}^{2}$. Multiplying the (coordinates of) points in this sphere by $e^{i \theta}$ creates a foliation of $S^{3}$ into circles. Collapsing each of these circles to a point yields a space diffeomorphic to $S^{2}$. It is 
well-known that if we equip $S^{3}$ with the round metric of radius 1 , we obtain a well-defined metric induced on $S^{2}$, namely the round metric with radius $\frac{1}{2}$. (See for example $[\mathbf{2} ;$ p. 6].)

Allowing $\lambda$ to vary in the above computations is equivalent to rescaling the $S^{1}$ fibres in the total space of the Hopf fibration. Clearly, setting $\lambda=1$ gives the round metric of radius 1 . When $S^{3}$ is equipped with one of these metrics for some value of $\lambda$, it is known as a Berger sphere. Indeed, our calculations agree with those for Berger spheres in $[\mathbf{2} ;$ p. 81]. In particular, note that as $\lambda \rightarrow 0$, the only meaningful curvature formula we are left with in the limit is that for $K\left(\epsilon_{2}, \epsilon_{3}\right)$. Clearly, as $\lambda \rightarrow 0, K\left(\epsilon_{2}, \epsilon_{3}\right) \rightarrow 4$, which agrees with the sectional curavture of $S^{2}\left(\frac{1}{2}\right)$.

Note further that we will have some zero curvatures when $\lambda=\frac{4}{3}$, and negative curvatures when $\lambda>\frac{4}{3}$. It may at first be surprising that a homogeneous metric on a sphere can display negative curvatures, given that the classic example of a homogeneous metric (the round metric) is also the classic example of a metric with positive curvature.

For the record, elementary further calculations show that

$$
\operatorname{Ric}\left(\epsilon_{1}\right)=2 \lambda, \quad \operatorname{Ric}\left(\epsilon_{2}\right)=4-2 \lambda, \quad \operatorname{Ric}\left(\epsilon_{3}\right)=4-2 \lambda ;
$$

and scal $=8-2 \lambda$.

We now turn our attention to our final example:

$$
\frac{S U(3)}{S U(2)} \cong S^{5}
$$

Think of $S^{5}$ as the unit sphere in $\mathbb{C}^{3}$. The transitive action of $S U(3)$ on $S^{5}$ is again the natural action. The isotropy group at the point $(1,0,0) \in \mathbb{C}^{3}$ is the subgroup of $S U(3)$ consisting of matrices taking the form

$$
\left(\begin{array}{ll}
1 & \\
& S U(2)
\end{array}\right) \text {. }
$$

This subgroup is clearly canonically isomorphic to $S U(2)$.

We can identify $T_{(1,0,0)} S^{5}$ with $\left\{\left(\operatorname{Re} z_{1}, z_{2}, z_{3}\right)\right\}$. Clearly, the isotropy representation fixes the line $\left(\operatorname{Re} z_{1}, 0,0\right)$. The subspace $\left\{\left(0, z_{2}, z_{3}\right)\right\}$ is also invariant and irreducible (as the natural action of $S U(2)$ on $\mathbb{C}^{2}$ is transitive on any distance sphere). Thus this tangent space splits as a direct sum of two irreducible representations. Up 
to scaling, we have a unique invariant inner product on each subspace. Moreover, as these representations are clearly inequivalent, the subspaces must be orthogonal with respect to any invariant inner product on the whole tangent space. Thus there is a 2-parameter family of isotropy-invariant inner products on $T_{(1,0,0)} S^{5}$, and hence a 2-parameter family of homogeneous metrics on $\frac{S U(3)}{S U(2)}$.

Recall now that any homogeneous metric on a homogeneous space $G / H$ can be regarded as being induced by a certain left-invariant metric on $G$. With this in mind we will 'lift' the problem of identifying the homogeneous metrics on $\frac{S U(3)}{S U(2)}$ to a study of left-invariant metrics on $S U(3)$.

The tangent space of $S U(3)$ at the identity (in other words the Lie algebra $s u(3)$ ) can be identified with the (real) vector space of three-by-three skew-hermitian matrices with zero trace. A basis is given by

$$
\begin{gathered}
v_{1}=\left(\begin{array}{rrr}
2 i & 0 & 0 \\
0 & -i & 0 \\
0 & 0 & -i
\end{array}\right) \quad v_{2}=\left(\begin{array}{rrr}
0 & 1 & 0 \\
-1 & 0 & 0 \\
0 & 0 & 0
\end{array}\right) \quad v_{3}=\left(\begin{array}{lll}
0 & i & 0 \\
i & 0 & 0 \\
0 & 0 & 0
\end{array}\right) \\
v_{4}=\left(\begin{array}{rrr}
0 & 0 & 1 \\
0 & 0 & 0 \\
-1 & 0 & 0
\end{array}\right) \quad v_{5}=\left(\begin{array}{lll}
0 & 0 & i \\
0 & 0 & 0 \\
i & 0 & 0
\end{array}\right) \quad v_{6}=\left(\begin{array}{rrr}
0 & 0 & 0 \\
0 & i & 0 \\
0 & 0 & -i
\end{array}\right) \\
v_{7}=\left(\begin{array}{rrr}
0 & 0 & 0 \\
0 & 0 & 1 \\
0 & -1 & 0
\end{array}\right) \quad v_{8}=\left(\begin{array}{lll}
0 & 0 & 0 \\
0 & 0 & i \\
0 & i & 0
\end{array}\right) .
\end{gathered}
$$

Notice that $\left\{v_{6}, v_{7}, v_{8}\right\}$ form a basis for the subspace of $s u(3)$ tangent to $S U(2)$ viewed as $\left(\begin{array}{ll}1 & \\ & S U(2)\end{array}\right) \subset S U(3)$. Henceforth this subspace will be denoted $s u(2)$. It is easily checked that $v_{1}$ is $\operatorname{Ad}_{S U(2)}$-invariant, as is the space spanned by $\left\{v_{2}, v_{3}, v_{4}, v_{5}\right\}$. Note that $\mathrm{Ad}_{S U(2)}$-invariance in $s u(3)$ corresponds to isotropy-invariance in the homogeneous space under the quotient map

$$
\pi_{*}: S U(3) \rightarrow \frac{S U(3)}{S U(2)}
$$


so $\pi_{*} \operatorname{Span}\left\{v_{1}\right\}$ and $\pi_{*} \operatorname{Span}\left\{v_{2}, v_{3}, v_{4}, v_{5}\right\}$ are the isotropy irreducible subspaces identified above.

On $s u(3)$ the expression $\langle A, B\rangle=-\operatorname{tr}(A B)$ defines a positive definite inner product. (This is clear as $\langle$,$\rangle is a scaling of the Killing$ form for $s u(3)$, and $S U(3)$ is compact and semi-simple.) The above basis is orthogonal with respect to this inner product. Furthermore, the restriction of $\langle$,$\rangle to \operatorname{Span}\left\{v_{1}, \ldots, v_{5}\right\}$ is $\operatorname{Ad}_{S U(2)}$-invariant. (This follows from the invariance of the Killing form under $\operatorname{Ad}_{S U(3)}$.) Thus the left-invariant metric $g$ on $S U(3)$ determined by $\langle$,$\rangle induces a$ well-defined homogeneous metric $\bar{g}$ on $\frac{S U(3)}{S U(2)}$. We also know that any other homogeneous metric differs from $\bar{g}$ at the distinguished coset by positive scaling factors on the two isotropy-irreducible subspaces $\pi_{*} \operatorname{Span}\left\{v_{1}\right\}$ and $\pi_{*} \operatorname{Span}\left\{v_{2}, v_{3}, v_{4}, v_{5}\right\}$.

Putting all this together, we see that given any $\mu_{1}, \mu_{2}>0$, the inner product $\langle,\rangle_{\mu_{1}, \mu_{2}}$ on $s u(3)$ defined on the basis $\left\{v_{i}\right\}$ by

$$
\left\langle v_{i}, v_{j}\right\rangle_{\mu_{1}, \mu_{2}}=\left\{\begin{array}{c}
\mu_{1}\left\langle v_{1}, v_{1}\right\rangle \text { if } i=j=1 \\
\mu_{2}\left\langle v_{i}, v_{j}\right\rangle \text { if } i, j \in\{2,3,4,5\} \\
\left\langle v_{i}, v_{j}\right\rangle \text { otherwise }
\end{array}\right.
$$

determines a left-invariant metric $g_{\mu_{1}, \mu_{2}}$ on $S U(3)$. This metric induces a well-defined homogeneous metric $\bar{g}_{\mu_{1}, \mu 2}$ on $\frac{S U(3)}{S U(2)}$. Moreover, every homogeneous metric on this space can be obtained in this way for some $\mu_{1}, \mu_{2}>0$.

We now turn to computing the curvature of the $\bar{g}_{\mu_{1}, \mu_{2}}$. As a first step, we consider the curvature of the left-invariant metrics determined by the $g_{\mu_{1}, \mu_{2}}$ on $S U(3)$. In fact it suffices to consider the curvature of the left-invariant metrics $g_{\lambda}$ on $S U(3)$ determined by the inner product

$$
\left\langle v_{i}, v_{j}\right\rangle_{\lambda}=\left\{\begin{array}{c}
\frac{\lambda}{2}\left\langle v_{1}, v_{1}\right\rangle \text { if } i=j=1 \\
\frac{1}{2}\left\langle v_{i}, v_{j}\right\rangle \text { otherwise }
\end{array}\right.
$$

where $\lambda$ is a positive constant. It is easy to see that any homogoneous metric on $\frac{S U(3)}{S U(2)}$ differs from one induced by a $g_{\lambda}$ by a global scaling 
factor. Recall that performing a global rescale of a Riemannian metric by a factor $k$ results in a scaling of the sectional curvatures by $\frac{1}{k}$. Therefore the essential features of the sectional curvatures displayed by the $\bar{g}_{\mu_{1}, \mu_{2}}$ will not be lost by making this simplification.

We define a new basis for $s u(3)$ as follows. Let $w_{1}=\frac{1}{\sqrt{3 \lambda}} v_{1}$, and set $w_{i}=v_{i}$ otherwise. This basis is orthonormal for $g_{\lambda}$, so we can use our left-invariant curvature formulas to compute the sectional curvatures of the resulting metric on $S U(3)$. In order to compute curvatures for $\bar{g}_{\lambda}$, we invoke the fact that $\pi:\left(S U(3), g_{\lambda}\right) \rightarrow\left(\frac{S U(3)}{S U(2)}, \bar{g}_{\lambda}\right)$ is a Riemannian submersion. The O'Neill formulas for a Riemannian submersion tells us that for $i, j \in\{1, \ldots, 5\}$ :

$$
K_{\bar{g}_{\lambda}}\left(\pi_{*}\left(w_{i}\right), \pi_{*}\left(w_{j}\right)\right)=K_{g_{\lambda}}\left(w_{i}, w_{j}\right)+\frac{3}{4}\left|\phi\left[w_{i}, w_{j}\right]\right|^{2}
$$

where $\phi$ denotes orthogonal projection onto

$$
s u(2)=\operatorname{Span}\left\{w_{6}, w_{7}, w_{8}\right\} .
$$

We obtain the following results:

$$
\begin{aligned}
& K_{\bar{g}_{\lambda}}\left(\pi_{*}\left(w_{1}\right), \pi_{*}\left(w_{j}\right)\right)=\frac{3}{4} \lambda \text { for } j=2,3,4,5 ; \\
& K_{\bar{g}_{\lambda}}\left(\pi_{*}\left(w_{i}\right), \pi_{*}\left(w_{j}\right)\right)=1 \text { for } i=2,3 \quad j=4,5 ; \\
& K_{\bar{g}_{\lambda}}\left(\pi_{*}\left(w_{i}\right), \pi_{*}\left(w_{j}\right)\right)=4-\frac{9}{4} \lambda \text { for }(i, j)=(2,3) \text { or }(4,5) .
\end{aligned}
$$

Let us analyse these expressions. First of all, notice that setting $\lambda=1$ gives a homogeneous metric of positive sectional curature - but not the round metric. The radius 1 round metric this time corresponds to $\lambda=\frac{4}{3}$. For $\lambda=\frac{16}{9}$ we get some zero curvatures, and for $\lambda>\frac{16}{9}$ we have some negative curvatures. Of course, it is clear that we can never get a homogeneous metric with everywhere zero or negative sectional curvatures for this space. (Investigating the limit as $\lambda \rightarrow 0$ gives information about curvatures of $\mathbb{C} P^{2}$.)

For the record, we also have $\operatorname{Ric}\left(\pi_{*}\left(w_{1}\right)\right)=3 \lambda$ and $\operatorname{Ric}\left(\pi_{*}\left(w_{i}\right)\right)=$ $6-\frac{3}{2} \lambda$ for $i=2,3,4,5$. The scalar curvature has the constant value $24-3 \lambda$. This concludes our study of homogeneous metrics on spheres. 
The authors would like to thank Jürgen Berndt, Johnny Burns, Michael Clancy and John Murray for conversations related to this work. The first author would also like to acknowledge the support of an IRCSET grant from the Irish government.

\section{REFERENCES}

[1] A. Besse, Einstein Manifolds, Springer-Verlag, 1987.

[2] P. Petersen, Riemannian Geometry, Springer-Verlag, 1998.

Martin Kerin and David Wraith,

Department of Mathematics,

National University of Ireland,

Maynooth, Ireland

dwraith@maths.may.ie

Received 24 March 2003. 